\title{
MicroRNA profiling of gastric cancer patients from formalin-fixed paraffin-embedded samples
}

\author{
SOSHI OSAWA ${ }^{1}$, YUTAKA SHIMADA ${ }^{1}$, SHINICHI SEKINE ${ }^{1}$, TOMOYUKI OKUMURA ${ }^{1}$, \\ TAKUYA NAGATA ${ }^{1}$, JUNYA FUKUOKA ${ }^{2}$ and KAZUHIRO TSUKADA ${ }^{1}$ \\ Department of ${ }^{1}$ Surgery and Science and ${ }^{2}$ Pathology, Graduate School of Medicine and \\ Pharmaceutical Sciences for Research, University of Toyama, Toyama, Japan
}

Received January 12, 2011; Accepted April 1, 2011

DOI: $10.3892 / \mathrm{ol} .2011 .313$

\begin{abstract}
MicroRNA (miRNA) is a small non-coding RNA that targets specific mRNA. Recent progress in the extraction of RNA from formalin-fixed paraffin-embedded (FFPE) tissues has facilitated miRNA profiling using samples stored in laboratories worldwide. In the present study, miRNA profiling of gastric cancer patients is determined using FFPE samples. First, criteria were established for determining evaluable RNA from the FFPE samples. miRNA profiling was then undertaken using miRNA oligo chips with 885 featured genes. The FFPE samples were obtained from 47 gastric cancer patients who underwent operations between 1997 and 2007. Results showed that out of 47 paired samples, 37 pairs (78.8\%) were evaluable by our criteria. A total of 30 miRNAs were significantly up-regulated and 11 miRNAs were down-regulated in gastric cancer compared with those in normal gastric tissue. Among these, 14 miRNAs, including miR-21, were identified as prognostic factors of gastric cancer patients. Furthermore, miR-34a was selected as an independent prognostic factor. In conclusion, we identified miRNAs that are associated with the prognosis of gastric cancer patients. miRNA profiling using FFPE samples is a useful and promising method of evaluation for samples stored in laboratories worldwide, and can generate extremely valuable clinical data.
\end{abstract}

\section{Introduction}

MicroRNAs (miRNAs) are a broad class of small, non-coding RNAs that negatively regulate protein expression. miRNAs can post-transcriptionally regulate the expression of hundreds of their target genes, thereby controlling a wide range of biolo-

Correspondence to: Dr Soshi Osawa, Department of Surgery and Science, Graduate School of Medicine and Pharmaceutical Sciences for Research, University of Toyama, 2630 Sugitani, Toyama 930-0194, Japan

E-mail: sosawa@med.u-toyama.ac.jp

Key words: formalin-fixed paraffin-embedded samples, prognosis, miRNA profiling gical functions such as cellular proliferation, differentiation and apoptosis (1). The expression of miRNAs was shown to be temporally and spatially regulated, whereas the disruption of miRNA physiological expression patterns was associated with a number of examples of human tumorigenesis, suggesting that they play a role as a novel class of oncogenes or tumor suppressor genes (2).

miRNA expression profiles were shown to be potential tools for cancer diagnosis and prediction of prognosis. Various miRNAs were reported to be associated with the clinical outcome of chronic lymphocytic leukemia (2), lung adenocarcinoma $(3,4)$, breast cancer $(5)$ and pancreatic cancers $(6,7)$. However, whether a miRNA signature can predict the clinical outcomes of gastric cancer has yet to be determined.

Formalin-fixed, paraffin-embedded (FFPE) tissue samples are an invaluable source for the study of human disease. A large number of the tissue blocks are archived worldwide with corresponding well-documented clinical histories and histopathological reports. The potential value of these archives for retrospective molecular studies has been well-recognized (8).

In this study, miRNA expression profiles from FFPE samples in gastric cancer were examined and compared with clinicopathological factors.

\section{Materials and methods}

Patients and tissue specimens. FFPE specimens of gastric cancer and associated patient information were collected from gastrointestinal surgery at Toyama University Hospital, Japan. A total of 47 specimens were obtained from 398 cancer patients who had undergone operations between 1997 and 2007. The specimens were fixed in formalin for less than 10 days and excluded stage I and IV patients in non-radical surgery (Fig. 1).

Pretreatment of FFPE specimens prior to RNA extraction. First, we examined the tumor ratio in FFPE blocks and then compared the miRNA expression in the tumor with that in normal tissue. Our preliminary study showed that a tumor occupancy cut-off value of $70 \%$ should be used for tumors in this study. FFPE blocks were cut vertically into thin sections, which were then sliced into horizontal sections. Samples of 10- $\mu \mathrm{m}$ horizontal slices were used (Fig. 2). 


\section{Total gastric cancer patients who underwent operations from 1997 to 2007} $\mathrm{N}=398$

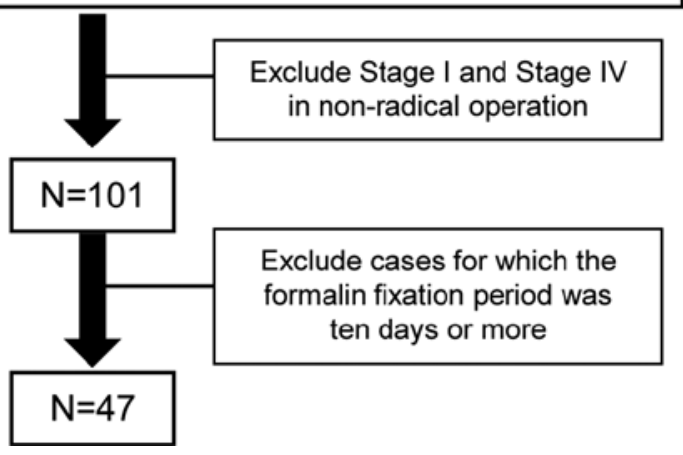

Figure 1. Selection of the gastric cancer patients in this study.
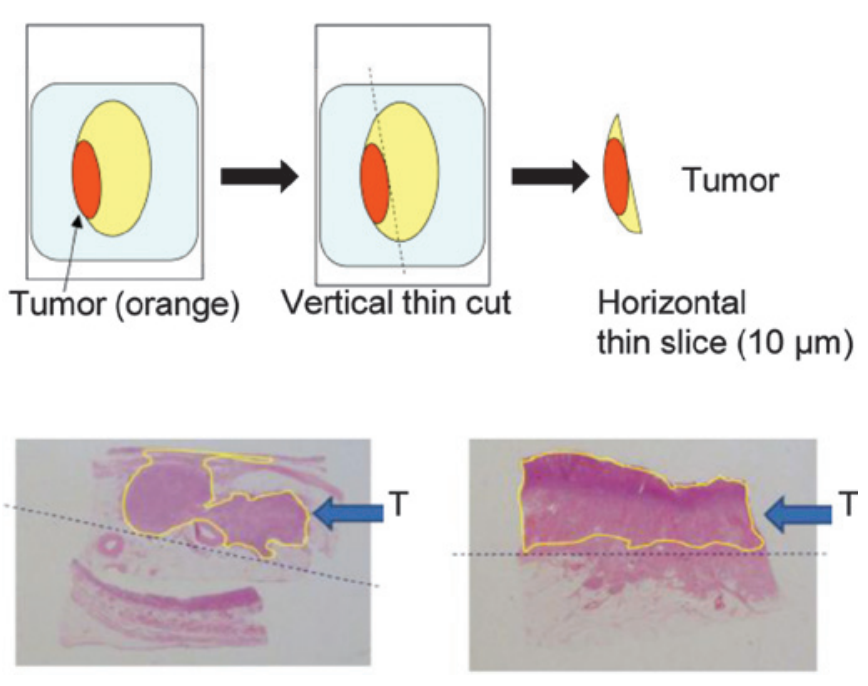

Figure 2. Method of making thin slices from a FFPE block. Tumor samples that occupy more than $70 \%$ of the slice tissue were used. $T$ indicates the tumor in the lower figures.

RNA extraction. Sections $(10-\mu \mathrm{m})$ were prepared from each FFPE specimen. Paraffin was removed by xylene treatment and tissues were washed with ethanol twice to remove xylene. Tissues were then treated with proteinase $\mathrm{K}$ at $37^{\circ} \mathrm{C}$ overnight. Following centrifugation, the supernatant was processed with a silica-based spin column (Toray Industries, Japan) in order to obtain purified total RNA. The degrees of RNA cross-linking and RNA degradation were analyzed by electrophoresis using an Agilent 2100 Bioanalyzer (Agilent Technologies, Santa Clara, CA, USA).

To estimate the possibility of analysis of RNA extracted from FFPE, selection criteria for RNA quality were applied. The RNA electrophoresis pattern was found to be crucial for estimation of the RNA quality for DNA microarray analysis. When the majority of RNAs were $>4000$ nucleotides in size due to cross-linking or when almost all of the RNAs were fragmented (e.g., <1000 nucleotides), the RNA quality was considered to be unsuitable for the miRNA analysis. On the
Table I. Clinical characteristics of the evaluable gastric cancer patients.

\begin{tabular}{lll}
\hline Characteristic & $\begin{array}{c}\text { No. of } \\
\text { patients }\end{array}$ & $\%$ \\
\hline
\end{tabular}

\begin{tabular}{lll}
\hline Age (yrs) & & \\
$<65$ & 10 & 27.0 \\
$\geq 65$ & 27 & 73.0 \\
Gender & & \\
Female & 11 & 29.7 \\
Male & 26 & 70.3
\end{tabular}

Tumor site

Lower third $\quad 13 \quad 35.1$

Middle third $\quad 11 \quad 29.8$

Upper third $\quad 10 \quad 27.0$

Entire

38.1

Tumor diameter (mm)

$$
\begin{aligned}
& \leq 20 \\
& >20 \text { and } \leq 50 \\
& >50 \text { and } \leq 100 \\
& >100
\end{aligned}
$$$$
5
$$

$\begin{array}{ll}13 & 35.1 \\ 15 & 40.5\end{array}$

$4 \quad 10.8$

Histological type

Differentiated $^{\mathrm{a}}$

Non-differentiated $^{\mathrm{b}}$

18

48.6

Depth of invasion

T1

T2

T3

T4

19

51.4

Lymph node metastasis

$\begin{array}{lrr}\text { Absent } & 7 & 18.9 \\ \text { Present } & 30 & 81.1\end{array}$

No. of involved lymph nodes

$\leq 6 \quad 16$

$\geq 7$

Adjuvant chemotherapy

Absent

14

37.8

Present

23

62.2

Operation method

Distal

21

56.8

Total

16

43.2

aDifferentiated type: well-differentiated, moderately differentiated, papillary. ${ }^{b}$ Non-differentiated type: poorly differentiated, signet-ring cell, mucinous.

basis of these criteria, we determined whether RNAs extracted from FFPE could be used for microarray analysis.

miRNA assays. miRNA profiling was examined using a Toray 3D-Gene ${ }^{\circledR}$ miRNA oligo chip (Toray Industries), which 
Table II. Over-expression of miRNAs in gastric cancers.

\begin{tabular}{|c|c|c|c|c|}
\hline ID & Normal & Tumor & $\mathrm{T} / \mathrm{N}$ ratio & P-value \\
\hline hsa-miR-185 & 15.6 & 23.9 & 1.53 & 0.0001 \\
\hline hsa-miR-106b & 20.6 & 50.1 & 2.44 & 0.0002 \\
\hline hsa-miR-17 & 48.7 & 88.2 & 1.81 & 0.0002 \\
\hline hsa-miR-425 & 17.9 & 31.6 & 1.76 & 0.0003 \\
\hline hsa-miR-106a & 28.7 & 53.4 & 1.86 & 0.0007 \\
\hline hsa-miR-20a & 13.9 & 28.5 & 2.04 & 0.0010 \\
\hline hsa-miR-181b & 10.1 & 15.3 & 1.51 & 0.0016 \\
\hline hsa-miR-25 & 43.2 & 71.2 & 1.65 & 0.0016 \\
\hline hsa-miR-93 & 52.6 & 91.4 & 1.74 & 0.0019 \\
\hline hsa-miR-21 & 76.3 & 208.6 & 2.73 & 0.0020 \\
\hline hsa-miR-20b & 11.8 & 21.5 & 1.82 & 0.0022 \\
\hline hsa-miR-192 & 104.7 & 205.4 & 1.96 & 0.0032 \\
\hline hsa-miR-130b & 4.7 & 7.2 & 1.55 & 0.0033 \\
\hline hsa-miR-146b-5p & 25.3 & 46.4 & 1.83 & 0.0035 \\
\hline hsa-miR-103 & 163.4 & 228.7 & 1.40 & 0.0054 \\
\hline hsa-miR-200a & 46.9 & 93.8 & 2.00 & 0.0069 \\
\hline hsa-miR-107 & 129.6 & 182.3 & 1.41 & 0.0075 \\
\hline hsa-miR-96 & 3.9 & 6.2 & 1.59 & 0.0076 \\
\hline hsa-miR-1308 & 2697.8 & 4333.4 & 1.61 & 0.0087 \\
\hline hsa-miR-222 & 58.5 & 88.9 & 1.52 & 0.0104 \\
\hline hsa-miR-194 & 152.4 & 300.8 & 1.97 & 0.0117 \\
\hline hsa-miR-16 & 133.6 & 199.8 & 1.50 & 0.0131 \\
\hline hsa-miR-1290 & 33.1 & 52.4 & 1.58 & 0.0151 \\
\hline hsa-miR-19b & 11.7 & 26.8 & 2.30 & 0.0153 \\
\hline hsa-miR-362-5p & 5.4 & 8.6 & 1.59 & 0.0173 \\
\hline hsa-miR-223 & 34.6 & 67.0 & 1.94 & 0.0204 \\
\hline hsa-miR-337-5p & 3.5 & 6.4 & 1.85 & 0.0205 \\
\hline hsa-miR-15a & 11.9 & 18.7 & 1.57 & 0.0216 \\
\hline hsa-miR-141 & 13.2 & 22.6 & 1.71 & 0.0358 \\
\hline hsa-miR-224 & 4.1 & 12.5 & 3.01 & 0.0497 \\
\hline hsa-miR-34a & 59.1 & 73.7 & 1.25 & $0.0935^{\mathrm{a}}$ \\
\hline
\end{tabular}

${ }^{a} \mathrm{~A}$ total of 30 miRNAs, with the exception of has-miR-34a, were significantly over-expressed in gastric cancer compared with those in normal gastric tissue $(\mathrm{P}<0.05)$.

is a DNA chip. The number of mounted genes on a chip is 885 in total.

RNA extracted from a sample was processed into an appropriate form. A solution adjusted for the DNA chip was applied (including $500 \mathrm{ng}$ of total RNA) and hybridization was performed. A probe complementary to the target nucleic acids was present on the DNA chip. The probe forms a double-stranded structure with the complementary target nucleic acids in the sample solution. The excess reagents were removed by washing and double strands formed by hybridization were detected. Of note is that double strands cannot be verified macroscopically. Therefore, a typical method involves fluorescent dye incorporation and the use of a detector to observe fluorescence. In this study, the expression level of
Table III. Reduced expression of miRNAs in gastric cancers.

\begin{tabular}{|c|c|c|c|c|}
\hline ID & Normal & Tumor & $\mathrm{T} / \mathrm{N}$ ratio & P-value \\
\hline hsa-miR-133a & 53.1 & 21.2 & 0.40 & 0.0000 \\
\hline hsa-miR-143 ${ }^{a}$ & 34.6 & 18.2 & 0.53 & 0.0000 \\
\hline hsa-miR-133b & 80.9 & 33.9 & 0.42 & 0.0000 \\
\hline hsa-miR-145 & 5925.8 & 2578.6 & 0.44 & 0.0000 \\
\hline hsa-miR-187 & 20.9 & 13.1 & 0.63 & 0.0002 \\
\hline hsa-miR-302b & 35.9 & 18.1 & 0.50 & 0.0024 \\
\hline hsa-miR-29c ${ }^{a}$ & 20.5 & 13.3 & 0.65 & 0.0031 \\
\hline hsa-miR-143 & 2024.1 & 1271.2 & 0.63 & 0.0127 \\
\hline hsa-miR-548m & 8.0 & 3.6 & 0.45 & 0.0139 \\
\hline hsa-miR-29a $a^{a}$ & 4.7 & 3.0 & 0.64 & 0.0264 \\
\hline hsa-let-7f-1 ${ }^{\text {a }}$ & 7.2 & 6.2 & 0.85 & 0.0345 \\
\hline
\end{tabular}

${ }^{\mathrm{a}} \mathrm{A}$ total of 11 miRNAs exhibited a significantly reduced expression in gastric cancer $(\mathrm{P}<0.05)$.

each miRNA was normalized using the median of signal strength of the entire gene in each chip.

$q R T-P C R$. cDNA was prepared from miRNA samples using a Taq Man microRNA reverse transcription kit on the ABI Prism ${ }^{\circledR} 7000$ real-time PCR system according to the manufacturer's instructions (Applied Biosystems, Foster City, CA, USA). Predesigned Taq Man microRNA assays for hsa-miR-21, hsa-miR-106b, hsa-miR-133a, hsa-miR-133b and hsa-miR-145 were purchased from Applied Biosystems. qRT-PCR was performed using a Taq Man universal PCR master mix, according to the manufacturer's protocol (Applied Biosystems). Each miRNA expression in FFPE specimens was estimated with a standard curve using human gastric reference RNA (Human stomach tumor total RNA, BD, No. 636629).

Statistical analysis. The expression levels of miRNA in tumor and normal tissues were analyzed by the t-test. The overall survival time was calculated from the date of operation until the patient succumbed to the disease or the last follow-up contact. The Kaplan-Meier method was used to estimate survival. The differences in survival for over-expression $(\mathrm{T} / \mathrm{N}$ ratio $>2.0)$ and reduced expression $(\mathrm{T} / \mathrm{N}$ ratio $<0.5)$ were analyzed using the log-rank test. A multivariate Cox regression analysis was utilized. Characteristics such as gender, age, TNM classification, histology, adjuvant chemotherapy and specific microRNA were used to investigate whether the microRNA signature is an independent predictor of overall survival in gastric cancer patients. Statistical analyses were conducted using Dr. SPSS II for Windows and JMP 8. Two-tailed tests and $\mathrm{P}<0.05$ were used for statistical significance.

\section{Results}

Out of 47 paired samples, 37 pairs $(78.8 \%)$ were evaluable by quality check. Table I shows the characteristics of the gastric cancer patients in the inclusion criteria. The patients of stages III and IV received post-operative 5FU-based adjuvant 
A

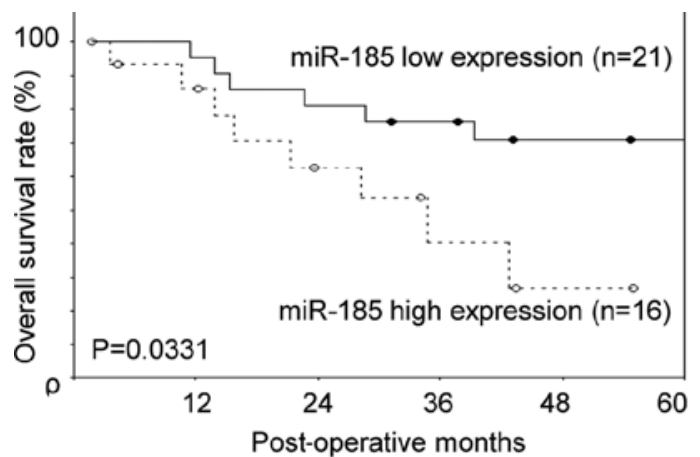

C

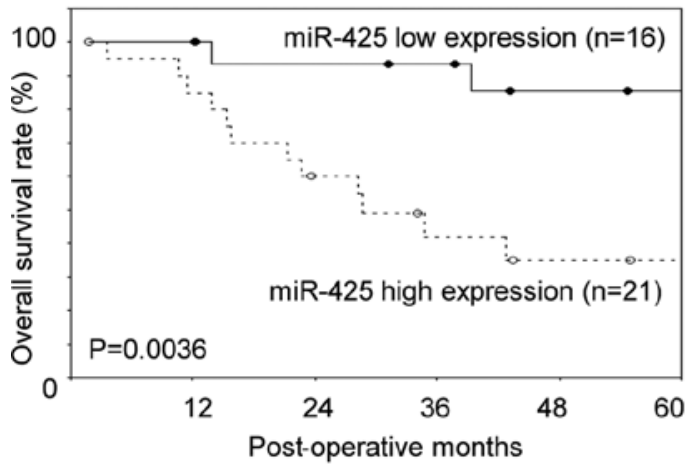

$\mathbf{E}$

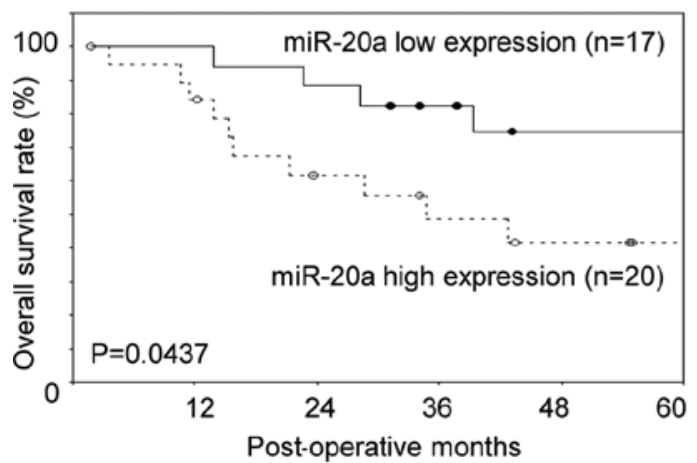

G

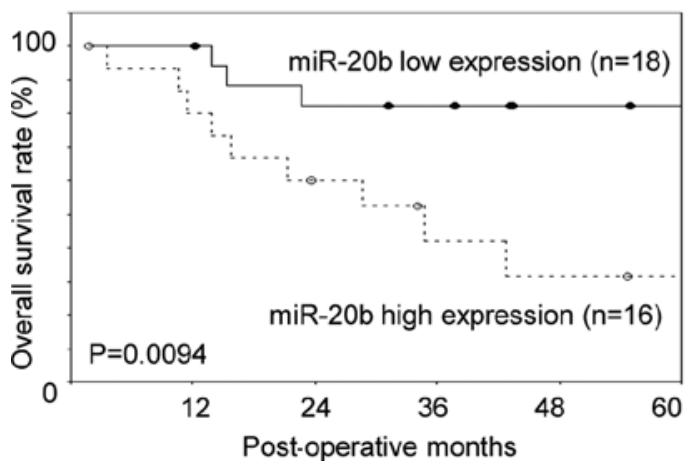

I

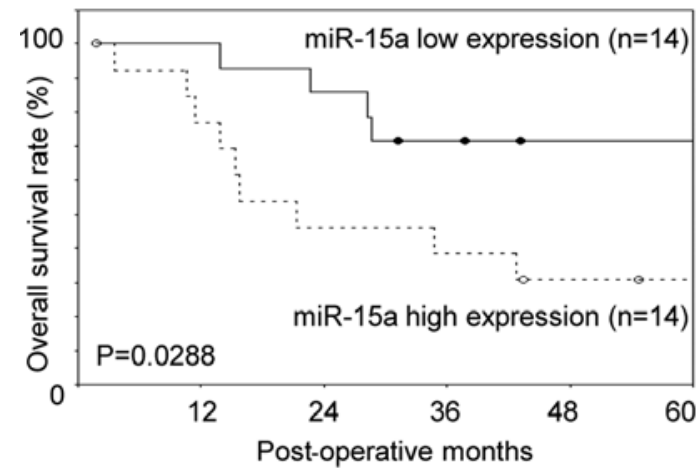

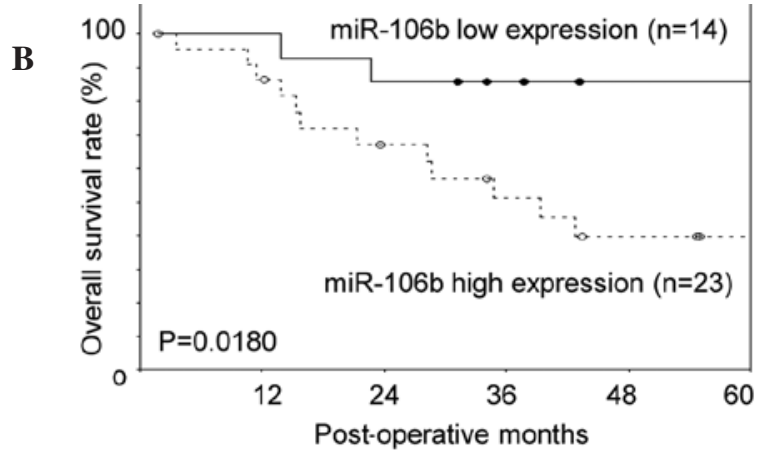
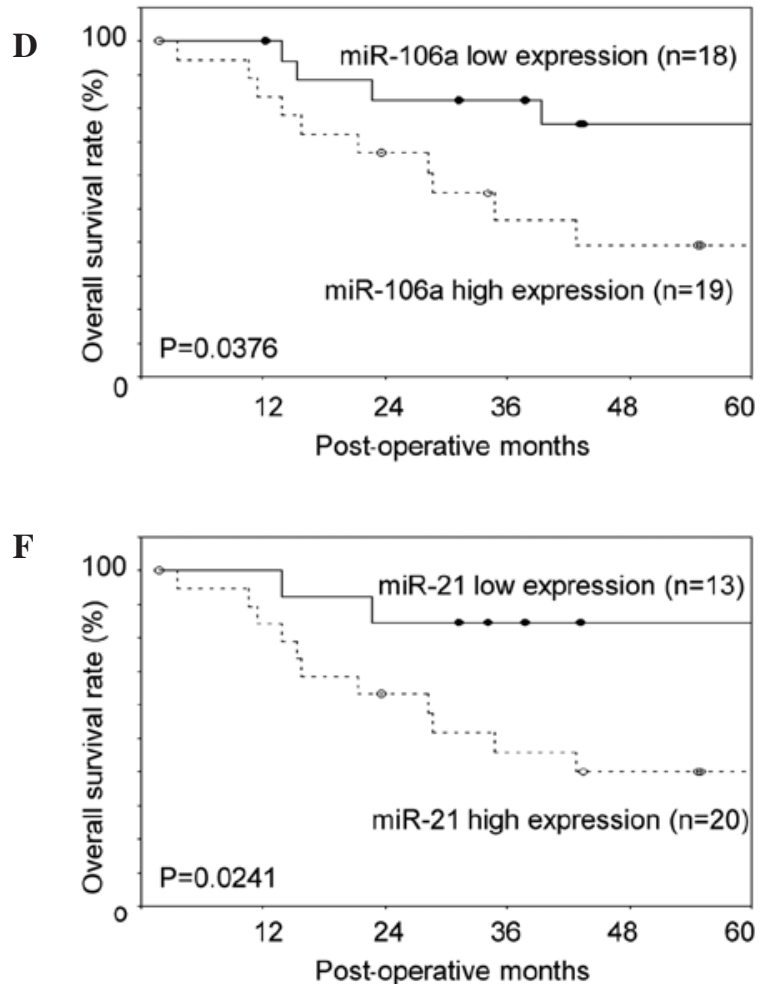

$\mathbf{H}$

$\mathbf{K}$

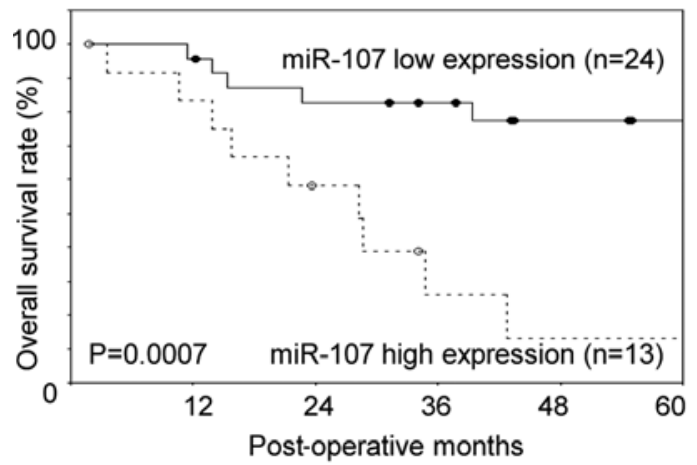

M

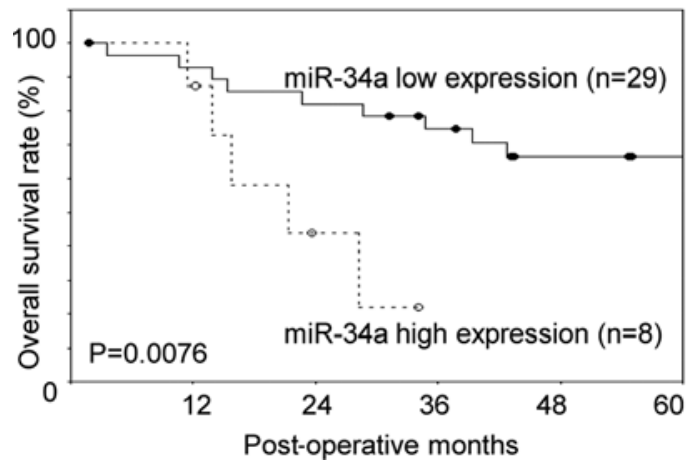

$\mathbf{L}$

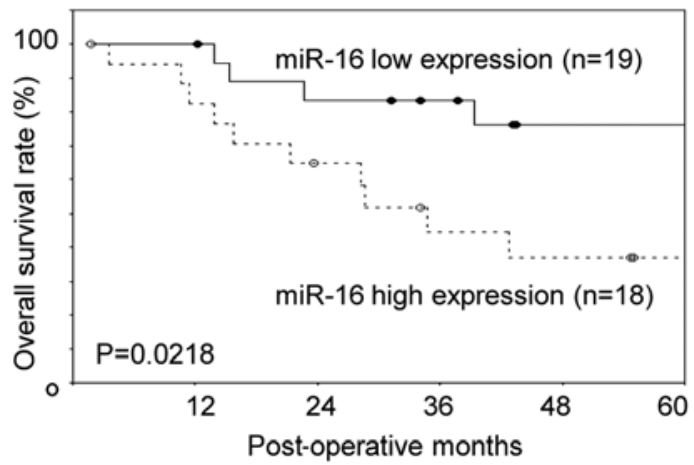

$\mathbf{N}$

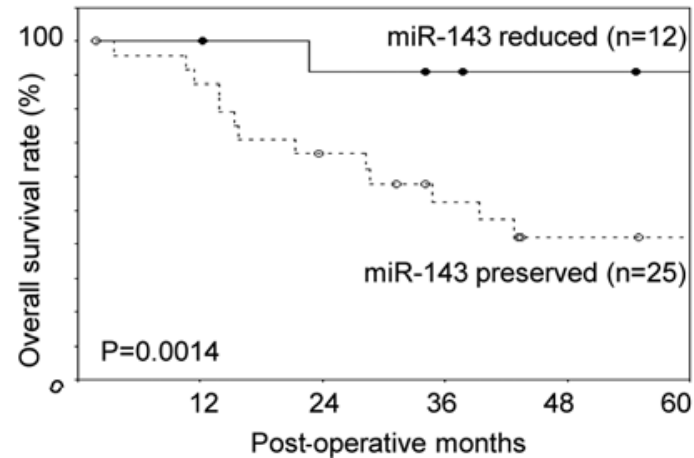

Figure 3. Survival curves of the patients. In total, 13 over-expressed miRNAs were found to be significant prognostic factors by Kaplan-Meier estimates of overall survival $(\mathrm{P}<0.05)$; all cases are shown. On the other hand, miR-143 was the only significant prognostic factor exhibiting a reduced miRNA expression.

chemotherapy and half of the patients of stage II received the same chemotherapy. Patients did not receive adjuvant radiotherapy.

Selection of specific miRNAs in gastric cancer. A total of 30 miRNAs were significantly over-expressed (T/N ratio $>1.40$ ) in gastric cancer compared with those in normal gastric tissue (Table II). On the other hand, the expressions of 11 miRNAs were significantly reduced $(\mathrm{T} / \mathrm{N}$ ratio $<0.85)$ in gastric cancer compared with those in normal gastric tissue (Table III).

Correlation with clinicopathological characteristics. In total, 13 over-expressed miRNAs were found to be significant prognostic factors by the Kaplan-Meier estimates of overall survival (median follow-up time, 37.8 months). These miRNAs were: miR-185, miR-106b, miR-425, miR-106a, miR-20a, miR-21, miR-20b, miR-200a, miR-15a, miR-103, miR-107, miR-16 and miR-34a. Fig. 3 shows the Kaplan-Meier estimates of overall survival and the 13 over-expressed miRNAs. Although the $\mathrm{T} / \mathrm{N}$ ratio of miR-34a expression was 1.25 , a significant difference in the Kaplan-Meier estimates of overall survival associated with its expression was observed $(\mathrm{P}=0.0076)$. On the other hand, miR-143 was the only significant prognostic factor exhibiting a reduced miRNA expression (Fig. 3). A multivariate Cox proportional hazard model revealed miR-34a to be an independent prognostic factor [risk ratio (RR) 7.11]. The prognostic factors are shown in Table IV.

qRT-PCR in FFPE specimens. miRNA expression was verified in the three remaining FFPE specimens using qRT-PCR. In FFPE specimens, the over-expression of miR-21 and
miR106b in DNA chips tended to increase, while a reduced expression was noted for miR-133a, miR-133b and miR-145 in DNA chips (Fig. 4).

\section{Discussion}

miRNA expression patterns have been described in various hematological and solid cancers (5,9-11). Findings of the present study showed that 14 miRNAs were associated with the prognosis of gastric cancer patients. Among these miRNAs, miR-21 and miR-20b have already been described in this context in the literature (9,12-14).

Antiapoptotic miR-21 is up-regulated in gastric cancer and is related to tumor growth $(9,12)$. miR-21 targets programmed cell death 4 (PDCD4) and maspin (SERPINB5), resulting in tumor invasion and metastasis.

miR-20b has been reported to accumulate in tumor cells and is considered to have an oncogenic role. It plays a crucial role in fine-tuning the adaptation of tumor cells to oxygen concentration. The inhibition of miR-20b was found to increase the protein levels of HIF-1a and VEGF in normoxic tumor cells (13) and it was one of the most highly expressed miRNAs in gastric cancer tissues (14).

In this study, the results of the multivariate Cox proportional hazard model showed that only miR-34a was an independent prognostic factor. In addition, miR-185 and miR-425 tended to have an independent prognostic impact on patient survival ( $R R$ 2.84, $\mathrm{P}=0.09$ and $\mathrm{RR} 4.88, \mathrm{P}=0.07$, respectively).

miR-34a is a well-known miRNA. It was identified as a p53 target by Welch et al (15), who reported that ectopic 
Table IV. miRNAs that were associated with the prognosis of gastric cancer patients.

\begin{tabular}{|c|c|c|c|c|}
\hline \multirow[b]{2}{*}{ ID } & \multirow{2}{*}{$\frac{\text { Univariate analysis }}{\text { Log-rank (P-value) }}$} & \multicolumn{3}{|c|}{ Cox proportional hazard model } \\
\hline & & Risk ratio & $95 \% \mathrm{CI}$ & $\mathrm{P}$-value \\
\hline hsa-miR-107 & 0.0007 & 2.05 & $0.40-12.21$ & 0.392 \\
\hline hsa-miR-103 & 0.0008 & 2.57 & $0.66-11.47$ & 0.174 \\
\hline hsa-miR-143 & 0.0014 & 1.63 & $0.11-22.36$ & 0.712 \\
\hline hsa-miR-425 & 0.0036 & 4.80 & $0.80-38.36$ & 0.086 \\
\hline hsa-miR-34a & 0.0076 & 7.49 & $1.59-40.19$ & $0.011^{\mathrm{a}}$ \\
\hline hsa-miR-20b & 0.0094 & 1.93 & $0.42-11.36$ & 0.410 \\
\hline hsa-miR-200a & 0.0096 & 2.19 & $0.33-19.69$ & 0.422 \\
\hline hsa-miR-106b & 0.0180 & 1.60 & $0.21-14.39$ & 0.643 \\
\hline hsa-miR-16 & 0.0218 & 1.14 & $0.29-4.860$ & 0.847 \\
\hline hsa-miR-21 & 0.0241 & 1.44 & $0.18-14.99$ & 0.732 \\
\hline hsa-miR-15a & 0.0288 & 1.95 & $0.47-9.130$ & 0.357 \\
\hline hsa-miR-185 & 0.0331 & 2.88 & $0.84-10.61$ & 0.092 \\
\hline hsa-miR-106a & 0.0376 & 1.73 & $0.38-8.500$ & 0.481 \\
\hline hsa-miR-20a & 0.0437 & 1.11 & $0.20-7.140$ & 0.907 \\
\hline
\end{tabular}

${ }^{a}$ Only miR-34a was an independent prognostic factor.

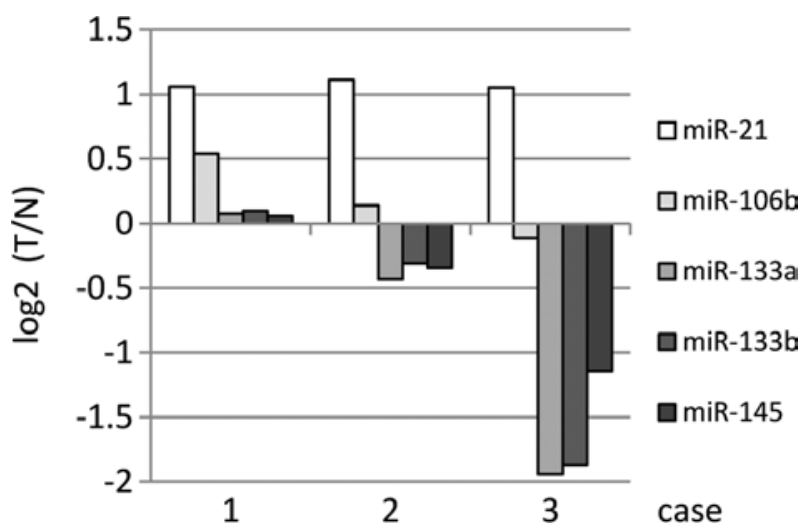

Figure 4. qRT-PCR using FFPE tissues. The expression of certain miRNAs was verified in the 3 remaining FFPE specimens by qRT-PCR. miR-21 and miR106b are over-expressed in DNA chips. miR-133a, miR-133b and miR-145 exhibit a reduced expression in DNA chips.

miR-34a induces apoptosis when reintroduced into neuroblastoma cell lines. In cancer, miR-34-mediated apoptosis may be suppressed by the inactivation of p53 and/or miR-34 genes. miR-34a was found to be expressed in hepatocellular carcinoma and colon cancer. In gastric carcinoma, Martin et al (16) reported that these carcinomas expressed high levels of p53 protein and survival analysis revealed a strong association between the p53 status of the tumor and patient survival time after diagnosis. In addition, Qing et al (17) showed that restoration of tumor suppressor miR-34 inhibits human p53-mutant gastric cancer tumorspheres.

A recent report (18) identified miRNAs differentially expressed in gastric carcinoma tissues. A total of 11 miRNAs were compatible with our results; however, miR-143, miR-200a and miR-185 were not listed.
In their study, Li et al (19) showed that a seven-microRNA signature (miR-10b, miR-21, miR-223, miR-338, let-7a, miR$30 a-5 p$ and miR-126) is closely associated with relapse-free and overall survival among patients with gastric cancer.

The results of our study showed for the first time that miR-34a is correlated with the prognosis of gastric cancer patients.

FFPE tissue samples are an invaluable source for the study of human disease. FFPE specimens are available in state hospitals and associated clinical data are usually recorded. These specimens are regarded as useful in the monitoring of diseases that have long-term clinical courses of treatment, such as breast and thyroid cancer. A large number of tissue blocks are archived worldwide with corresponding welldocumented clinical histories and histopathological reports. In addition, it was reported that there was a high correlation in miRNA expression between paired FFPE and fresh frozen material by quantitative RT-PCR (20). Although our data from microarray analysis do not completely correlate with the results of qRT-PCR from the remaining FFPE specimens, we believe that miRNA from FFPE may be a valuable source.

The experimental method established in this study may be useful for gene expression analysis in translational research. Although we detected various significant miRNAs in gastric cancer, the sample size was limited and definite conclusions could not be drawn. Moreover, we confirmed the relationships of these miRNAs to biological functions, such as cellular proliferation, invasion, chemosensitivity and lymph node metastasis, in gastric cancer.

In conclusion, our results identified miRNAs that are associated with prognosis in gastric cancer patients. miRNA profiling using FFPE samples is a useful and promising method of evaluating samples that are stored in laboratories worldwid and are accompanied by extremely valuable clinical data. 


\section{Acknowledgements}

The authors thank Drs Hiroyuki Takahashi and Yasuo Takano for their technical support.

\section{References}

1. Calin GA and Croce CM: MicroRNA signatures in human cancers. Nat Rev Cancer 6: 857-866, 2006.

2. Calin GA, Ferracin M, Cimmino A, et al: A microRNA signature associated with prognosis and progression in chronic lymphocytic leukemia. N Engl J Med 353: 1793-1801, 2005.

3. Yanaihara N, Caplen N, Bowman E, et al: Unique microRNA molecular profiles in lung cancer diagnosis and prognosis. Cancer Cell 9: 189-198, 2006.

4. Takamizawa J, Konishi H, Yanagisawa K, et al: Reduced expression of the let-7 microRNAs in human lung cancers in association with shortened postoperative survival. Cancer Res 64: 3753-3756, 2004

5. Iorio MV, Ferracin M, Liu CG, et al: MicroRNA gene expression deregulation in human breast cancer. Cancer Res 65: 7065-7070, 2005 .

6. Bloomston M, Frankel WL, Petrocca F, et al: MicroRNA expression patterns to differentiate pancreatic adenocarcinoma from normal pancreas and chronic pancreatitis. JAMA 297: 1901-1908, 2007.

7. Roldo C, Missiaglia E, Hagan JP, et al: MicroRNA expression abnormalities in pancreatic endocrine and acinar tumors are associated with distinctive pathologic features and clinical behavior. J Clin Oncol 24: 4677-4684, 2006.

8. Lewis F, Maughan NJ, Smith V, et al: Unlocking the archivegene expression in paraffin-embedded tissue. J Pathol 195 66-71, 2001.

9. Volinia S, Calin GA, Liu CG, et al: A microRNA expression signature of human solid tumors defines cancer gene targets. Proc Natl Acad Sci USA 103: 2257-2261, 2006.
10. Gramantieri L, Ferracin M, Fornari F, et al: Cyclin G1 is a target of miR-122a, a microRNA frequently down-regulated in human hepatocellular carcinoma. Cancer Res 67: 6092-6099, 2007.

11. Schetter AJ, Leung SY, Sohn JJ, et al: MicroRNA expression profiles associated with prognosis and therapeutic outcome in colon adenocarcinoma. JAMA 299: 425-436, 2008.

12. Zhang Z, Li Z, Gao C, et al: miR-21 plays a pivotal role in gastric cancer pathogenesis and progression. Lab Invest 88: 1358-1366, 2008.

13. Lei Z, Li B, Yang Z, et al: Regulation of HIF-1alpha and VEGF by miR-20b tunes tumor cells to adapt to the alteration of oxygen concentration. PLoS One 4: e7629, 2009.

14. Guo J, Miao Y, Xiao B, et al: Differential expression of microRNA species in human gastric cancer versus non-tumorous tissues. J Gastroenterol Hepatol 24: 652-657, 2009.

15. Welch C, Chen Y, Stallings RL: MicroRNA-34a functions as a potential tumor suppressor by inducing apoptosis in neuroblastoma cells. Oncogene 26: 5017-5022, 2007.

16. Martin HM, Filipe MI, Morris RW, et al: p53 expression and prognosis in gastric carcinoma. Int J Cancer 50: 859-862, 1992.

17. Qing Ji, Xinbao Hao, Yang Meng, et al: Restoration of tumor suppressor miR-34 inhibits human p53-mutant gastric cancer tumorspheres. BMC Cancer 8: 266, 2008.

18. Yoshiyuki T, Chisato N, Tsuyoshi N, et al: MicroRNA-375 is downregulated in gastric carcinomas and regulates cell survival by targeting PDK1 and 14-3-3\}. Cancer Res 70: 2339, 2010.

19. Li X, Zhang Y, Zhang Y, et al: Survival prediction of gastric cancer by a seven-microRNA signature. Gut 59: 579-585, 2010.

20. Glud M, Klausen M, Gniadecki R, et al: MicroRNA expression in melanocytic nevi: the usefulness of formalin-fixed, paraffinembedded material for miRNA microarray profiling. J Invest Dermatol 129: 1219-1224, 2009. 
